\title{
Estructura de la población y distribución de Anadara tuberculosa Sowerby (1833) (Mollusca: Bivalvia) en los manglares de Golfito y Playa Blanca de Puerto Jiménez, Golfo Dulce, Costa Rica
}

\author{
Ana Margarita Silva-Benavides ${ }^{1,2}$ \& Roger Bonilla ${ }^{3}$ \\ 1. Escuela de Biología, Universidad de Costa Rica, San Pedro, San José 2060, Costa Rica; ana.silva@ucr.ac.cr \\ 2. Centro de Investigación en Ciencias del Mar y Limnología (CIMAR), Ciudad de la Investigación, Universidad de \\ Costa Rica, San Pedro, San José 2060, Costa Rica; ana.silva@ucr.ac.cr \\ 3. Escuela de Estadística, Universidad de Costa Rica, San Pedro, San José 2060, Costa Rica; roger.bonilla@ucr.ac.cr
}

\author{
Recibido11-VIII-2014. C Corregido 20-XI-2014. Aceptado 22-XII-2014.
}

\begin{abstract}
Population structure and distribution of Anadara tuberculosa Sowerby (1833) (Mollusca: Bivalvia) in the mangroves of Golfito and Playa Blanca, Puerto Jiménez, Golfo Dulce, Costa Rica. The density, population, length, yield and sex proportion of the Anadara tuberculosa Sowerby (1833) were studied in the mangroves of Golfito and Playa Blanca, Golfo Dulce, Costa Rica from 2008-2010. A. tuberculosa was more abundant in Golfito mangroves $\left(2.77\right.$ units $\left.\mathrm{m}^{2}\right)$, than Playa Blanca mangrove $\left(0.14\right.$ units $\left.\mathrm{m}^{2}\right)$. The average lengths were $42.1 \mathrm{~mm}$ for Golfito (under the Costa Rican legal minimal length for exploitation: $47 \mathrm{~mm}$ ) and 48.35mm for Playa Blanca mangrove. Maximum densities in Golfito were measured in the outlet of Purruja River (mangrove of Purruja) and mangroves Congo, Ceibo y la Trocha. The average total weights were $24.48 \mathrm{~g}$ for Golfito and 36.35g for Playa Blanca. The condition index (a meat yield measurement) was higher in Golfito $(23.45 \%)$ than in Playa Blanca (17.01\%). The male ratio was 1:1. These results suggest that A. tuberculosa population is declining in these mangroves. Therefore, it's necessary to implement repopulation projects of the mollusc in the mangroves of Golfo Dulce, in conjunction with government authorities, NGOs and civil society. Mangrove workers and consumers are needed in that potential projects, to make the mollusc a sustainable natural resource exploited by different community members. Rev. Biol. Trop. 63 (Suppl. 1): 287-298. Epub 2015 April 01.
\end{abstract}

Key words: Anadara tuberculosa, mangrove, Mollusca, Golfo Dulce, Costa Rica, density, yield.

Los manglares de la costa Pacífica desde Baja California Sur, México hasta el Norte de Perú, constituyen un sitio de reproducción para un gran número de especies de bivalvos de importancia económica como Anadara tuberculosa Sowerby (1833) y A. similis Adams (1852) de la familia Arcidae (Squires, Esteves, Barona, \& Mora, 1975; MacKenzie, 2001; Manjarrés, Lucero, Gualteros, Cantera \& Gil, 2013); conocidas en Costa Rica como pianguas. Esta familia se distribuye en las zonas tropicales-subtropicales en substratos lodosos de los bosques de mangle y asociada principalmente a las raíces de las especies de
Rhizophora mangle y $R$. racemosa (Squires et al., 1975; Stern-Pirlot \& Wolff, 2006).

En Costa Rica, ambas especies constituyen una de las principales fuentes de ingreso económico por parte de los habitantes de los manglares de la costa del Pacífico Sur, a cuya extracción se han dedicado las comunidades desde hace varios años (Silva \& Carrillo, 2004; Morales, Silva, \& González, 2009; Morales, Silva, González, Villalobos, \& Ash, 2011; Pérez-Rubio, 2012). Sin embargo, estos organismos se han extraído de manera no controlada, situación que perjudica no solamente a los organismos per se, sino también a las personas 
que se han dedicado a la extracción de la piangua (llamados piangüeros). Debido a la importancia económica de estos bivalvos, es urgente diseñar un plan de manejo en los manglares del Golfo Dulce, y de esta manera garantizar la sobrevivencia de este recurso. Para esto, es fundamental obtener información biológica sobre características como la distribución, densidad, talla y rendimiento de la carne de esta especie de bivalvo en estos manglares.

Anadara tuberculosa y $A$. similis han sido objeto de varios estudios en la costa pacífica americana (Cruz, 1984; Vega, 1994; MacKenzie, 2001; Borda \& Cruz, 2004; Stern-Pirlot \& Wolff, 2006; Flores \& Licandeo, 2010; Lucero, Cantera, \& Neira, 2012; Lucero et al., 2013; Manjarrés, et al., 2013), donde se analizan aspectos relacionados con la biología, reclutamiento, densidad y ecología de ambas especies; cuyos resultados han servido como base para entender la dinámica poblacional de estos organismos y ofrecer medidas de protección de un recurso que se encuentra actualmente sobreexplotado en varios países de América.

El objetivo del presente estudio consiste en proporcionar información sobre la estructura poblacional de Anadara tuberculosa en los manglares de Golfito y Playa Blanca de Puerto Jiménez, Golfo Dulce, Costa Rica; cuya información puede ser utilizada para la toma de decisiones en un eventual plan de manejo para estos manglares.

\section{MATERIALES Y MÉTODOS}

Durante los años 2008, 2009 y 2010, se realizaron colectas mensuales de pianguas en 12 sitios de varios manglares de Golfito y siete sectores en el manglar de Playa Blanca de Puerto Jiménez, Golfo Dulce. (Fig. 1a, b). Los bivalvos se muestrearon en 25 cuadrantes en los manglares de Golfito (2008-2010) cuya área comprende $10 \times 10 \mathrm{~m}$ cada uno y en 14 cuadrantes con áreas de $5 \times 5 \mathrm{~m}$ en Playa Blanca (2009-2010). Del total de individuos capturados de $A$. tuberculosa en cada manglar se realizaron las siguientes mediciones: longitud de la valva en mm (con un calibrador Vernier $\pm 0.1 \mathrm{~mm}$ ), peso total y peso fresco en $\mathrm{g}$ (con una balanza OHAUS de $0,100 \mathrm{~g}$ de precisión) índice de condición de rendimiento (peso fresco de la carne/peso total *100) (Cruz \& Palacios, 1983). Se calculó la densidad de la población considerando el número de individuos y el área muestreada. La determinación del sexo se efectuó por medio de la observación directa con un microscopio Nikon eclipse Ci 022901. La salinidad (ups) del agua intersticial se midió con un refractómetro (Modelo RHS-10ATC) y los análisis granulométricos del suelo se realizaron según Ellison (1998).

Se efectuó un análisis de variancia (ANOVA) para comparar los grupos. Las pruebas de diferencias post-hoc se analizaron con la prueba de Tukey. El nivel de significancia $\alpha$ de las pruebas fue del 0.05 y se usó el paquete estadístico STATA para el procesamiento de la información (StataCorp, 2005).

\section{RESULTADOS}

Densidad: La diferencia de densidad entre los manglares es significativa $(\mathrm{z}=-5.34$, $\mathrm{p}=0.00$ ). En los manglares de Golfito se recolectaron 8683 ejemplares de A. tuberculosa; con una densidad promedio de 2.99ind. $/ \mathrm{m}^{2}$. El sitio con mayor densidad correspondió a la desembocadura del río Purruja en el manglar de Purruja (6ind. $/ \mathrm{m}^{2}$ ) y los de menor densidad pertenecen a los sitios El Cultivo (0.97ind./ $\mathrm{m}^{2}$ ) y Puntarenitas (0.96ind. $\left./ \mathrm{m}^{2}\right)$ (Cuadro 1$)$. En el manglar de Playa Blanca se recolectaron 175 ejemplares, con una densidad promedia de $0.14 \mathrm{ind} . / \mathrm{m}^{2}$. La mayor densidad se encontró en las zonas de muestreo aledañas al Río Timonel y la menor densidad se detectó en los sectores posteriores del manglar (Cuadro 2).

Longitud: Para los manglares de Golfito, el estudio indica que los individuos capturados presentaron una longitud promedio de $42.51 \pm 0.30 \mathrm{~mm}$. El intervalo de longitud comprendido entre 40 y $45 \mathrm{~mm}$ presentó el mayor número de individuos colectados. Es importante considerar la alta frecuencia de individuos en el intervalo de longitud entre 45 y $50 \mathrm{~mm}$. 

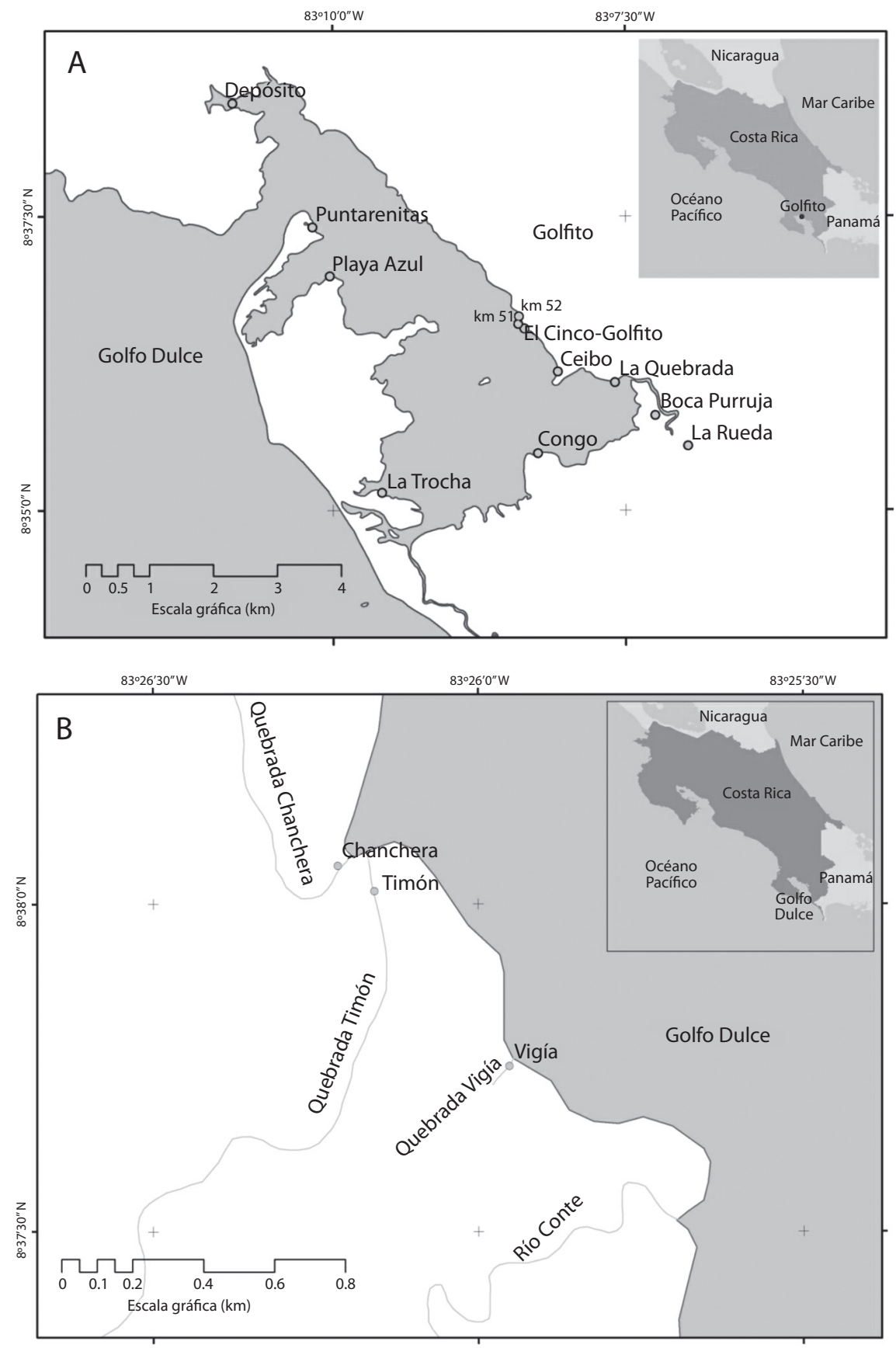

Fig. 1. Sitios de muestreo en (A) Golfito y en (B) Playa Blanca, Puerto Jiménez. Golfo Dulce, Costa Rica.

Fig. 1. Sampling sites: (A) Golfito and (B) Playa Blanca, Puerto Jimenez. Golfo Dulce, Costa Rica. 
CUADRO 1

Intervalos de confianza al 95\% de las variables biométricas de Anadara tuberculosa en Golfito, Golfo Dulce, Costa Rica

TABLE 1

Confidence intervals at $95 \%$ of biometric variables of Anadara tuberculosa in Golfito, Golfo Dulce, Costa Rica

\begin{tabular}{lccccc}
\multicolumn{1}{c}{ Sitios de muestreo } & Densidad $\left(\mathrm{ind} . / \mathrm{m}^{2}\right)$ & Longitud $(\mathrm{mm})$ & Peso total $(\mathrm{g})$ & Peso fresco $(\mathrm{g})$ & Rendimiento $(\%)$ \\
Golfito & 2.99 & $42.51 \pm 0.30$ & $24.48 \pm 0.49$ & $5.95 \pm 0.11$ & $23.45 \pm 0.40$ \\
$\begin{array}{l}\text { Desembocadura del Río Purruja, } \\
\text { manglar de Purruja. }\end{array}$ & 6.00 & $41.00 \pm 1.20$ & $20.99 \pm 2.10$ & $6.08 \pm 0.56$ & $29.59 \pm 0.93$ \\
Ceibo & & & & \\
Congo & 3.00 & $44.92 \pm 0.80$ & $30.25 \pm 2.40$ & $8.33 \pm 0.51$ & $28.41 \pm 1.20$ \\
Cultivo (sector posterior del & 3.71 & $40.30 \pm 0.51$ & $23.29 \pm 0.80$ & $5.69 \pm 0.20$ & $24.88 \pm 0.42$ \\
manglar de Purruja) & 0.97 & $40.93 \pm 1.82$ & $22.22 \pm 2.73$ & $4.28 \pm 0.47$ & $20.09 \pm 1.17$ \\
$\begin{array}{l}\text { Depósito (frente al centro } \\
\text { comercial el Depósito, Golfito) }\end{array}$ & 3.80 & $43.55 \pm 1.01$ & $25.33 \pm 1.83$ & $4.90 \pm 0.33$ & $20.39 \pm 0.99$ \\
Golfito Km 5 & & & & \\
La Quebrada & 2.18 & $43.56 \pm 1.02$ & $28.30 \pm 2.15$ & $4.48 \pm 0.31$ & $16.57 \pm 0.51$ \\
La Rueda (sector posterior del & 3.00 & $41.11 \pm 0.90$ & $18.47 \pm 1.19$ & $4.79 \pm 0.31$ & $26.25 \pm 0.69$ \\
manglar de Purruja) & 1.43 & $40.90 \pm 2.00$ & $24.62 \pm 2.67$ & $5.17 \pm 0.47$ & $22.36 \pm 1.10$ \\
La Trocha & & & & \\
Playa Azul & 4.40 & $44.40 \pm 0.60$ & $26.62 \pm 1.13$ & $6.09 \pm 0.29$ & $24.08 \pm 1.85$ \\
Puntarenitas & 2.35 & $45.91 \pm 1.21$ & $28.85 \pm 2.37$ & $5.99 \pm 0.47$ & $21.25 \pm 0.72$ \\
$\begin{array}{l}\text { Purruja La Isla (sector medio } \\
\text { del manglar de Purruja) }\end{array}$ & 0.96 & $44.30 \pm 1.32$ & $26.04 \pm 2.42$ & $4.55 \pm 0.43$ & $17.74 \pm 0.89$ \\
\hline
\end{tabular}

$\mathrm{n}$ Golfito $=8683$

CUADRO 2

Intervalos de confianza al 95\% de las variables biométricas de Anadara tuberculosa en Playa Blanca de Puerto Jiménez, Golfo Dulce, Costa Rica

TABLE 2

Confidence intervals at 95\% of biometric variables of Anadara tuberculosa in Playa Blanca, Puerto Jimenez, Golfo Dulce, Costa Rica

\begin{tabular}{lccccc}
\multicolumn{1}{c}{ Sitio de muestreo } & $\begin{array}{c}\text { Densidad } \\
\left(\mathrm{ind} . / \mathrm{m}^{2}\right)\end{array}$ & $\begin{array}{c}\text { Longitud } \\
(\mathrm{mm})\end{array}$ & $\begin{array}{c}\text { Peso total } \\
(\mathrm{g})\end{array}$ & $\begin{array}{c}\text { Peso fresco } \\
(\mathrm{g})\end{array}$ & $\begin{array}{c}\% \\
\text { Rendimiento }\end{array}$ \\
Playa Blanca & 0.14 & $48.35 \pm 0.9$ & $36.35 \pm 2.52$ & $6.09 \pm 0.41$ & $17.01 \pm 1.31$ \\
Q. Chanchera sector posterior del manglar & 0.08 & $50.47 \pm 11.12$ & $46.84 \pm 46.13$ & $4.49 \pm 3.07$ & $9.58 \pm 3.55$ \\
Q. Chanchera, sector medio del manglar & 0.11 & $44.21 \pm 4.33$ & $26.83 \pm 11.45$ & $4.55 \pm 1.66$ & $16.96 \pm 4.19$ \\
$\begin{array}{l}\text { R.Timonel, sector 1 } \\
\text { (margen derecha del R. Timonel) }\end{array}$ & 0.23 & $46.92 \pm 6.83$ & $35.16 \pm 13.82$ & $7.21 \pm 2.18$ & $20.50 \pm 9.52$ \\
R Timonel, sector 2 & 0.13 & $49.28 \pm 7.25$ & $35.92 \pm 14.57$ & $7.80 \pm 2.76$ & $21.71 \pm 5.49$ \\
(margen izquierda del R. Timonel). & & & & & \\
Afluente R. Timonel & 0.20 & $49.21 \pm 6.22$ & $40.66 \pm 17.31$ & $7.47 \pm 1.66$ & $18.37 \pm 5.32$ \\
Estero Vigía & 0.09 & $49.56 \pm 4.98$ & $33.01 \pm 17.50$ & $3.98 \pm 2.05$ & $12.05 \pm 6.74$ \\
Islote (sector posterior del manglar). & 0.095 & $48.82 \pm 5.21$ & $36.02 \pm 12.73$ & $7.18 \pm 1.95$ & $19.93 \pm 4.66$ \\
\hline
\end{tabular}

n Playa Blanca $=175$.

Tallas de longitud menores de $35 \mathrm{~mm}$ y mayores de $50 \mathrm{~mm}$ fueron poco evidentes (Fig. 2a). Aunque la talla de los individuos mantuvo una distribución relativamente homogénea durante el periodo de muestreo, se encontraron diferencias (ANOVA, $\mathrm{p}=0.00$ ) con respecto a los sitios de muestreo. El sector medio del manglar de Purruja (La Isla), mostró el menor tamaño (promedio $39.52 \pm 1.41 \mathrm{~mm}$ ), mientras que el manglar en Playa Azul presentó la máxima talla (45.90土1.21 mm). (Cuadro 1).

Para los sitios del manglar de Playa Blanca, el estudio indica que el mayor número de individuos capturados se encontraron entre las 

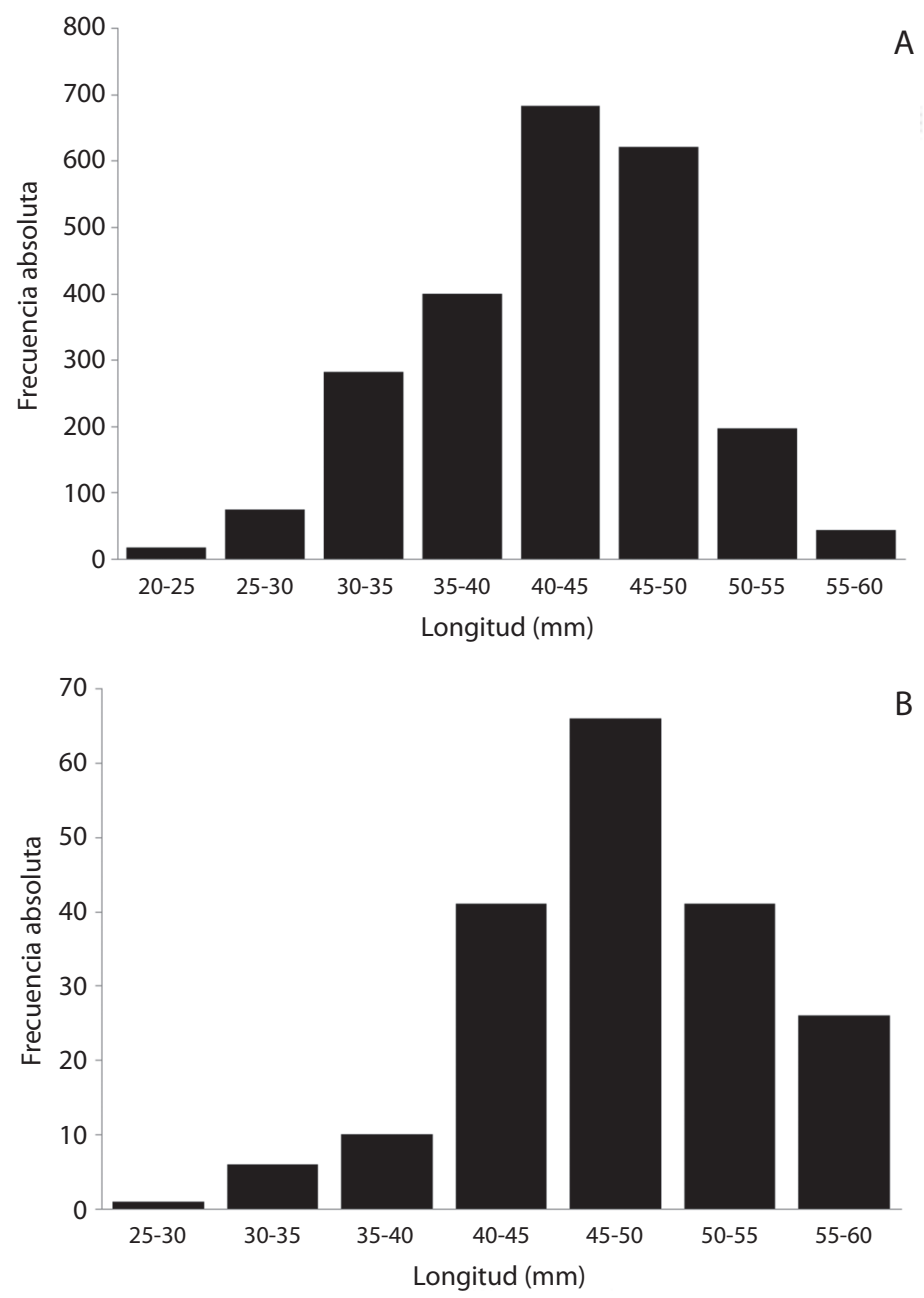

Fig. 2. Distribución de frecuencias de tallas de longitud de A. tuberculosa. (A) Golfito, (B) Playa Blanca de Puerto Jiménez. Golfo Dulce, Costa Rica.

Fig. 2. Length frequency distributions of A. tuberculosa. (A) Golfito, (B) Playa Blanca, Puerto Jiménez. Golfo Dulce, Costa Rica.

longitudes de 45 y $50 \mathrm{~mm}$, con un promedio de $48.35 \pm 0.90 \mathrm{~mm}$ (Fig. 2b).

Peso total: En los sectores del manglar de Playa Blanca, el peso total de A. tuberculosa presentó un promedio de $36.35 \pm 2.52 \mathrm{~g}$, significativamente mayor que en los manglares de Golfito (24.48 \pm 0.49 ) (ANOVA, $p=0.00)$. En todos los sitios muestreados en Golfito y Playa Blanca se observó una tendencia a aumentar el peso total, al incrementarse la longitud del animal (Fig. 3a, b).

Peso fresco: Los individuos capturados en Golfito presentaron un menor peso fresco que los individuos en los manglares de Playa Blanca (Cuadros 1 y 2), esa diferencia es significativa (ANOVA, $\mathrm{p}=0.001)$. Entre los manglares de Golfito, el Ceibo presentó el mayor peso fresco de todos los sitios muestreados $(8.33 \pm 0.51 \mathrm{~g})$. 

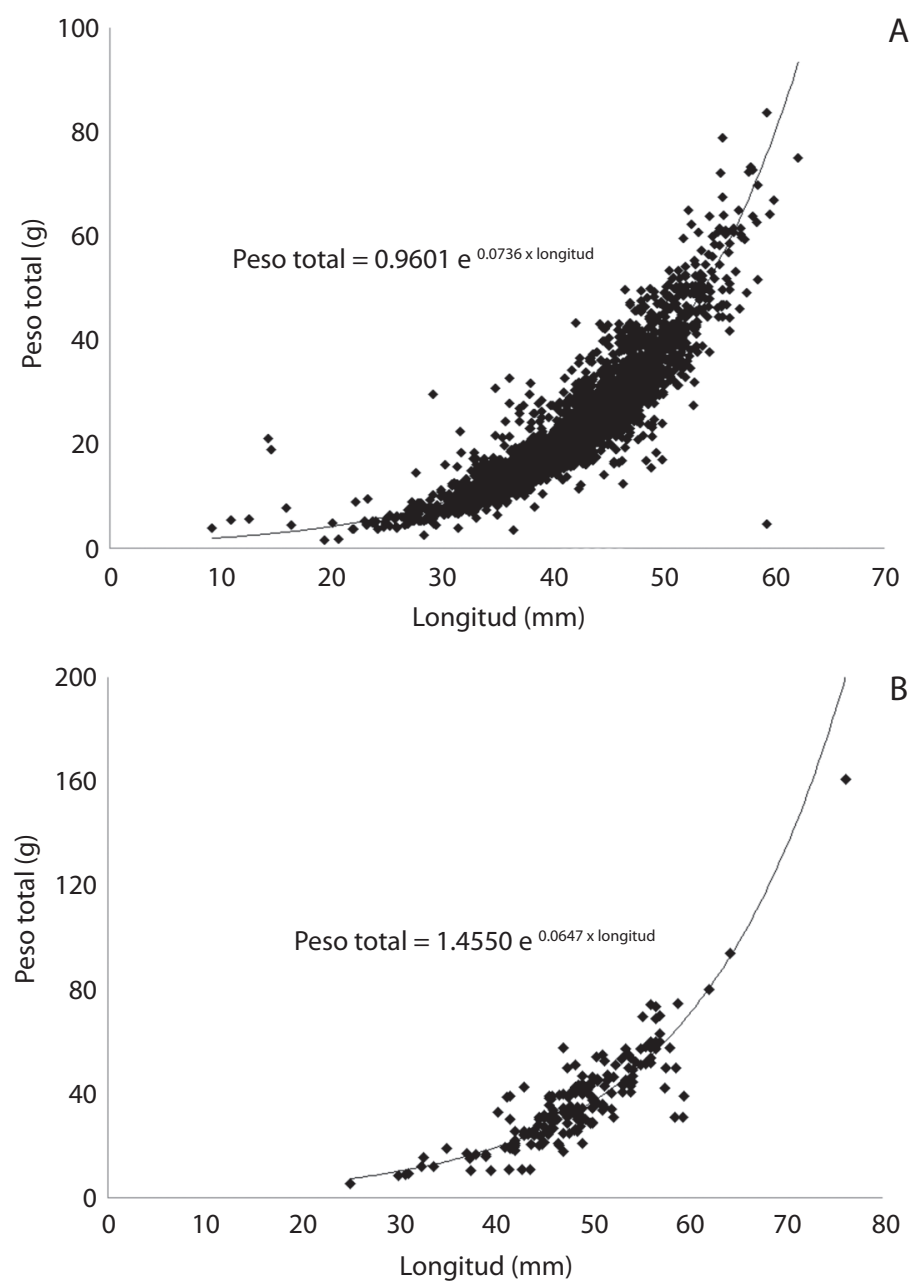

Fig. 3. Relación entre el peso total (g) y longitud (mm) de A. tuberculosa. (A) Golfito, (B) Playa Blanca de Puerto Jiménez. Golfo Dulce, Costa Rica.

Fig. 3. Relationship between total weight (g) and lenght (mm) of A. tuberculosa. (A) Golfito, (B) Playa Blanca, Puerto Jiménez. Golfo Dulce, Costa Rica.

Para los sitios del manglar de Playa Blanca, el análisis de variancia no presentó diferencia significativa entre los sitios de muestreo (ANOVA, $\mathrm{p}=0.315$ ), excepto para el sitio Vigía cuyo peso resultó menor que los otros lugares (Tukey, $\mathrm{p}=0.00$ ) (Cuadro 2).

Para todos los sitios muestreados, existe una relación positiva entre el peso fresco y longitud (Golfito $\mathrm{r}=0.7691$, $\mathrm{p}=0.00$; Playa Blanca $r=0.5908, p=0.00$ ) (Fig. 4a, b). Sin embargo, como se observa en la Fig. 5a y b, se presentó una relación inversa entre el peso total y peso fresco en ambas zonas de muestreo, lo que significa que un aumento en el peso total, no significa un aumento en el peso fresco del organismo.

Rendimiento: El porcentaje de rendimiento de la carne de A. tuberculosa en los manglares de Golfito resultó significativamente mayor $(23.45 \pm 0.40 \%)$ que en los sitios de recolecta del manglar de Playa Blanca $(17.01 \pm 1.31 \%)$ (ANOVA, $\mathrm{p}=0.00$ ). Entre los primeros, los sectores de Purruja, la Isla $(29.84 \pm 1.09)$ y la 

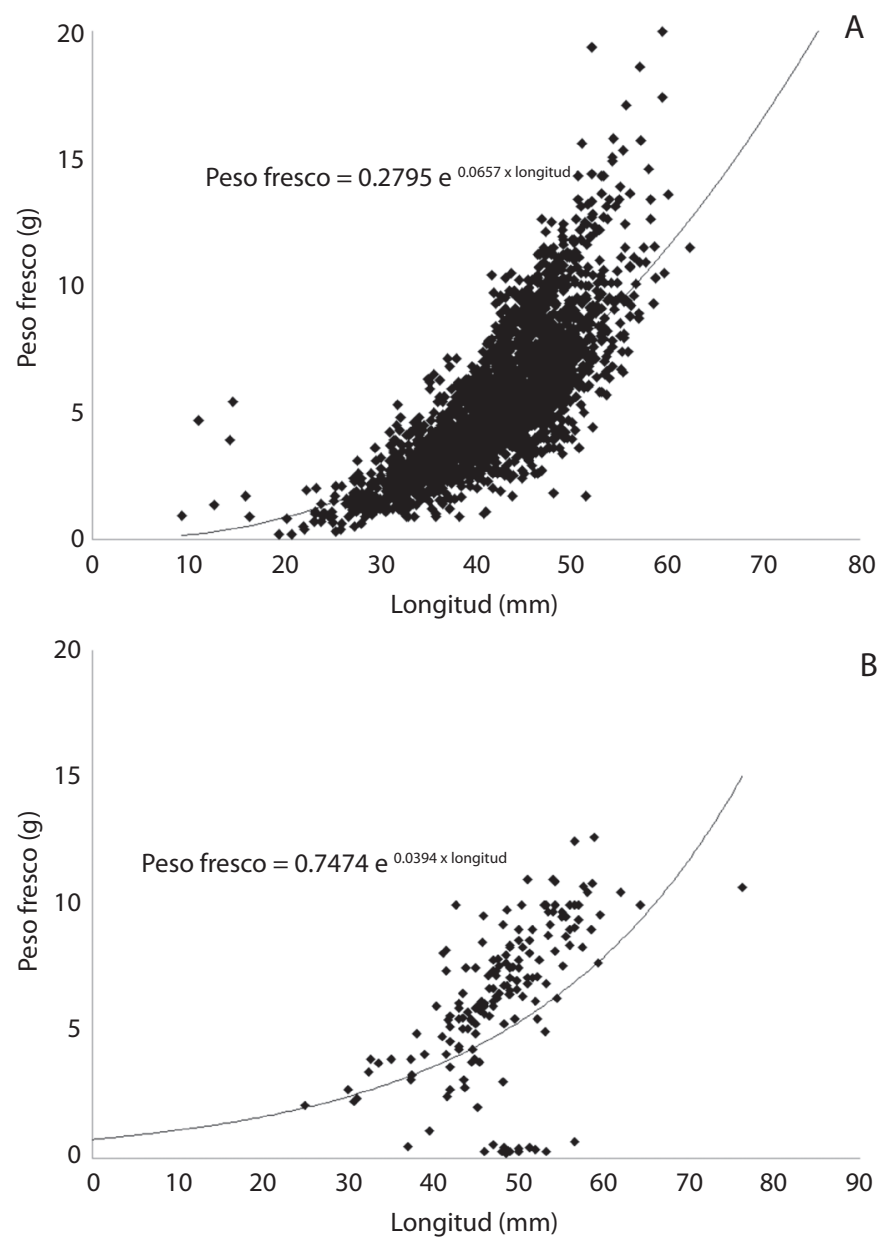

Fig. 4. Relación entre el peso fresco (g) y longitud (mm) de A. tuberculosa. (A) Golfito, (B) Playa Blanca de Puerto Jiménez. Golfo Dulce, Costa Rica.

Fig. 4. Relationship between wet weight (g) and lenght (mm) of A. tuberculosa. (A) Golfito, (B) Playa Blanca, Puerto Jiménez. Golfo Dulce, Costa Rica.

desembocadura del río Purruja (29.59 $\pm 0.93 \%)$, ambos en el manglar de Purruja, corresponden a los sitios que presentaron los mejores rendimientos (Cuadro 1). En el manglar de Playa Blanca, la margen izquierda y derecha del río Timonel, son los lugares que mostraron los valores más altos (Cuadro 2).

La Fig. 6a y b presenta la distribución del porcentaje del rendimiento con respecto a las diferentes clases de longitudes ( $\mathrm{mm}$ ) para todos los sitios muestreados. Para los manglares de Golfito, se observa un máximo rendimiento entre la longitud 25-30mm (Fig. 6a), mientras que para Playa Blanca, el máximo rendimiento se presentó en el intervalo de longitud entre 30-35mm (Fig. 6b).

Proporción de sexos: Los resultados muestran una proporción de sexos de 1:1 en todos los sitios de muestreo, $(\mathrm{p}>0.05)$.

Salinidad intersticial: Durante el periodo de estudio, la salinidad osciló entre 21 y 28 ups en los manglares de Golfito; presentándose los valores más bajos (21 ups) en el sector la Isla en el manglar del río Purruja y en el manglar 

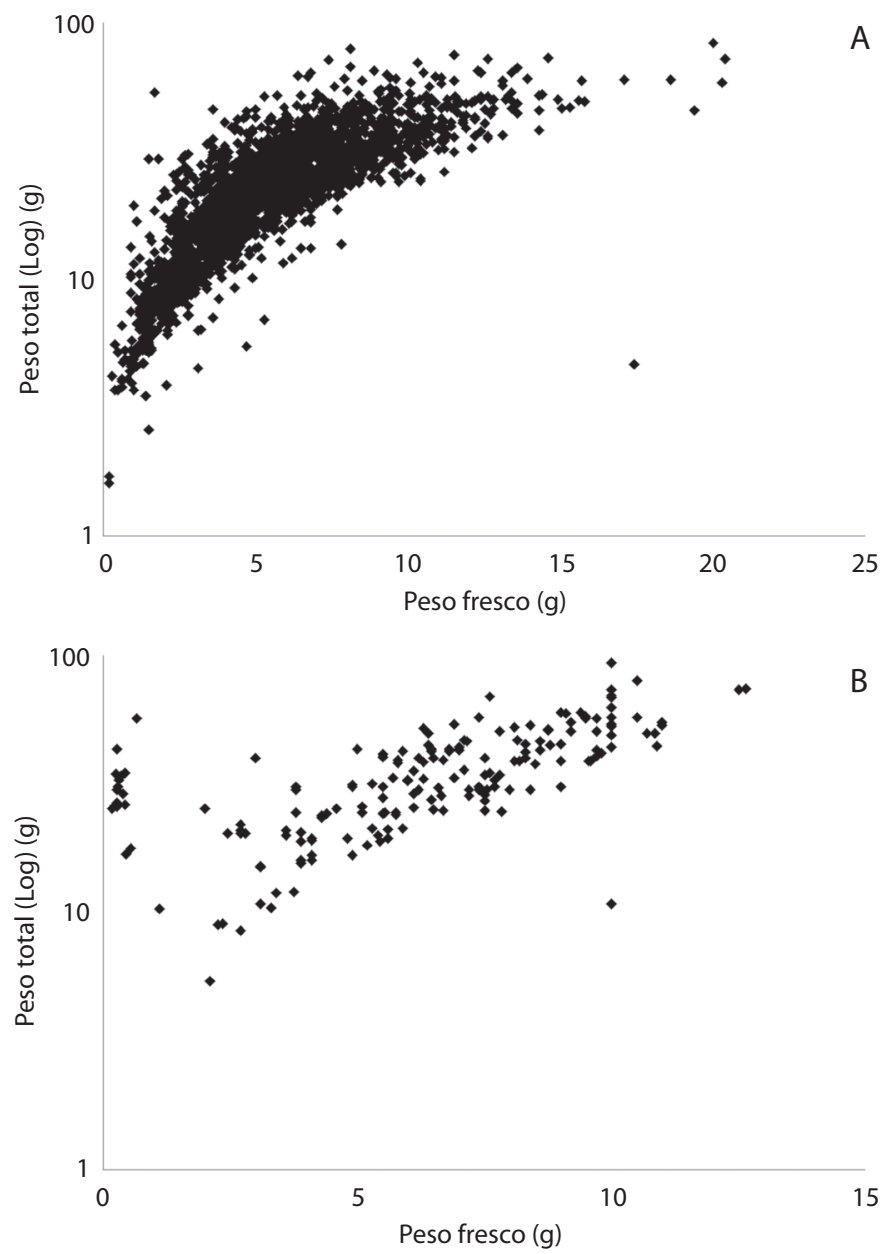

Fig. 5. Relación entre el peso total $(\log )(\mathrm{g})$ y el peso fresco (g) de A. tuberculosa. (A) Golfito, (B) Playa Blanca de Puerto Jiménez. Golfo Dulce, Costa Rica.

Fig. 5. Relationship between total weight (g) and dry weight (g) of A. tuberculosa. (A) Golfito, (B) Playa Blanca, Puerto Jiménez. Golfo Dulce, Costa Rica.

en el $\mathrm{Km}$ 5. Los valores más altos (28 ups) se reportan para el manglar el Congo. Para los sitios del manglar de Playa Blanca los valores fluctuaron entre 26 y 33 ups. Debido que no se presentó diferencias significativas entre los sitios de muestreo $(\mathrm{p}>0,05)$, esta variable no parece influir en la abundancia, ni en la distribución de los organismos en este estudio.

Análisis granulométrico: En Golfito, el análisis granulométrico resultó en un sedimento tipo lodo en todos los sitios de muestreo, solamente los sitios el Cultivo, la Rueda y
Puntarenitas se obtuvieron los mayores porcentajes en gravas y arenas. En Playa Blanca, los sustratos predominantes fueron las gravas y arenas en todos los sitios de muestreo.

\section{DISCUSIÓN}

En Costa Rica, la extracción de la piangua en los manglares constituye una fuente importante de ingreso económico para los habitantes que viven aledaños a las zonas de los manglares (Silva \& Carrillo, 2004; Pérez-Rubio, 

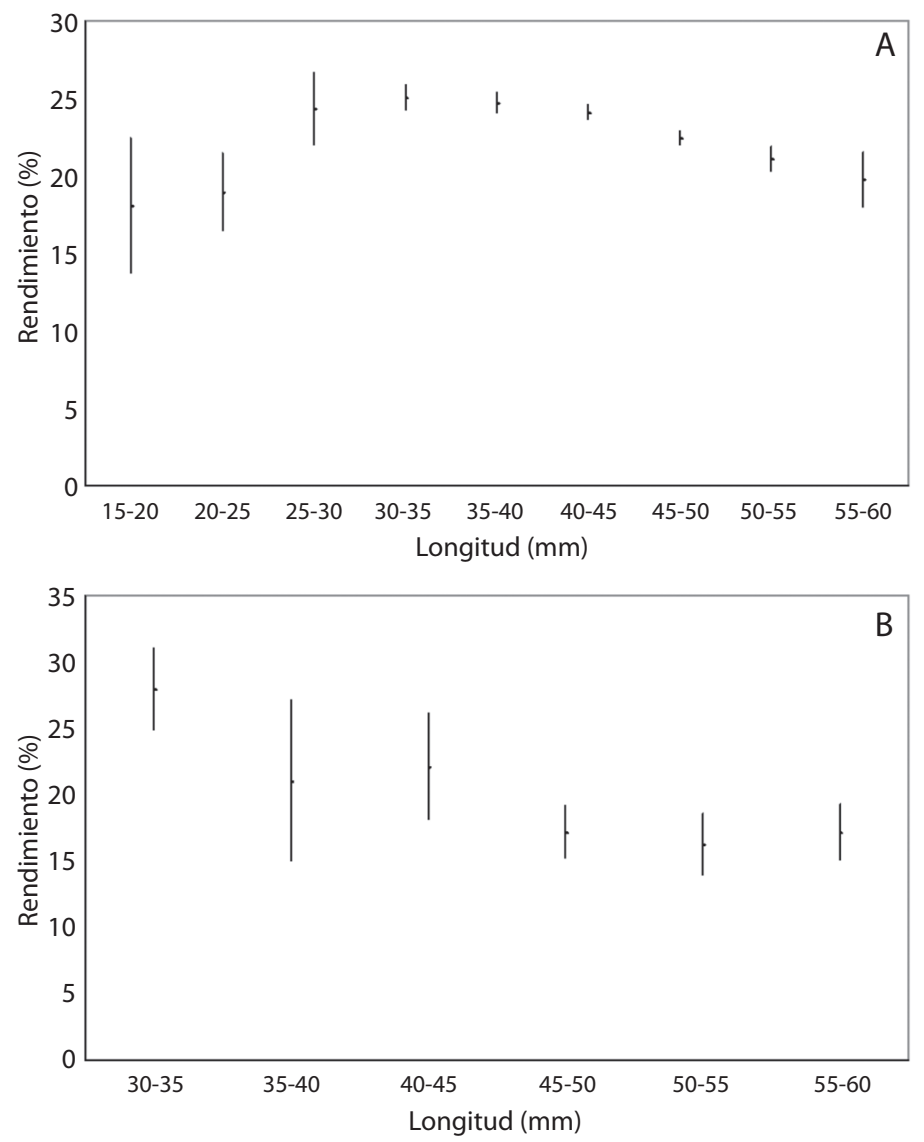

Fig. 6. Relación entre el rendimiento de la carne (\%) y longitud (mm) de A. tuberculosa. (A) Golfito, (B) Playa Blanca de Puerto Jiménez. Golfo Dulce, Costa Rica.

Fig. 6. Relationship between meat yield (\%) and lenght (mm) of A. tuberculosa. (A) Golfito, (B) Playa Blanca, Puerto Jiménez. Golfo Dulce, Costa Rica.

2012); por consiguiente, un plan de manejo de protección de esta especie por parte de las autoridades gubernamentales es importante para evitar la sobre-explotación de este bivalvo.

La baja densidad de A. tuberculosa en los manglares de Golfito, Playa Blanca y estudios previamente realizados en el manglar de Purruja, Golfito (Silva \& Bonilla, 2001; Pérez-Rubio, 2012), contrario a las mayores densidades de esta especie reportadas en manglares de México y Panamá (MacKenzie, 2001), permiten sugerir que un plan de protección es urgente para conservar un recurso como la piangua. Según Ampie y Cruz (1989) y De la Cruz (1994), A. tuberculosa inicia su madurez sexual en el intervalo de longitud de $23.2-26.2 \mathrm{~mm}$, alcanzando su tamaño comercial entre los 18 y 24 meses de edad, por lo cual se considera un recurso renovable comercial Lucero et al., (2012) y Lucero et al., (2013). Mencionan que las tallas medias de madurez sexual corresponden a 43.50 y $46.68 \mathrm{~mm}$ para esta especie en Colombia. En el presente estudio, no se midió la madurez sexual; sin embargo, si se considera como referencia los datos anteriores; en Costa Rica, la talla mínima legal de captura $(47 \mathrm{~mm})$ se encuentra cercana a las tallas medias de la madurez sexual mencionadas por estos autores, lo que podría indicar altos niveles de extracción del recurso. 
El presente estudio mostró una relación macho: hembra de 1:1, con individuos maduros presentes durante el periodo de análisis, que podría indicar que existe un desove continuo. Investigaciones realizadas por Cruz (1984) en Costa Rica y Lucero et al., (2013) en Colombia, mostraron máximos de desove entre los meses de mayo-setiembre y noviembre-marzo respectivamente. La presencia de individuos maduros podría indicar que la población se encuentra en reproducción continua durante todo el año, característica que no limitaría su crecimiento. Sin embargo, la alta intensidad en los niveles de extracción no permite que la mayoría de los individuos se encuentren cercanos a la talla de madurez mínima.

El manglar de Playa Blanca mostró una menor densidad de A. tuberculosa comparado con los manglares de Golfito; sin embargo, los individuos en Playa Blanca presentaron un mayor peso total y longitud (Cuadro 2). La baja densidad de pianguas que presentó el manglar de Playa Blanca, no representa una alternativa atractiva de captura para los piangueros; por consiguiente, la poca extracción favorece un mayor crecimiento de los organismos; mientras que en Golfito, la alta demanda por parte de los usuarios hacia este recurso, ejerce una presión sobre la población que no permite un crecimiento adecuado.

En tallas superiores, el rendimiento es menor, lo que indica un crecimiento de la concha pero no de las partes blandas, que se refleja en la incorporación de carbonato de calcio o partículas adheridas a la concha que inciden negativamente en el rendimiento, el cual es un indicador de la salud del animal (Cruz, 1982; De la Cruz, 1994; Manjarrés, 2011; Lucero et al., 2012; Lucero et al., 2013; Manjarrés et al., 2013). De manera similar a lo reportado por Silva y Bonilla (2001), el máximo rendimiento en todos los sitos de muestreo en Golfito se presentó en las longitudes comprendidas entre 25 y $30 \mathrm{~mm}$. En el caso de Playa Blanca, el mayor rendimiento se localiza entre las longitudes de 30 y $35 \mathrm{~mm}$, situación atribuida probablemente a la mayor longitud que presentan los individuos en este sitio.
La distribución de la piangua está relacionada con la textura y el tipo de suelo; por consiguiente, este organismo está restringido a substratos lodosos y a las raíces de Rhizophora mangle y $R$. racemosa (Vega, 1994; MacKenzie, 2001). Al comparar los sitios de muestreo en los manglares de Golfito y Playa Blanca, se observó mayor densidad en los bancos cuyo substrato mostró características lodosas y la presencia de raíces de $R$. mangle. Los sitios cercanos a las márgenes de los canales y cerca de las desembocaduras, presentan un substrato más suave que los sitios internos del manglar; condición que favorece el establecimiento de las pianguas como en la desembocadura del rio Purruja. También los manglares el Congo, Ceibo, la Trocha y el sector medio del manglar Purruja denominado la Isla, exhibieron las mayores densidades de $A$. tuberculosa; sitios que presentan una dominancia de substrato limoso-arcilloso. En el caso del manglar de Playa Blanca, existe una dominancia de gravas y arenas; situación similar se presentó en los sectores que se encuentran en la parte posterior del manglar de Purruja denominados el Cultivo y la Rueda y en el manglar de Puntarenitas en Golfito; lugares que presentaron menor densidad de pianguas.

En Costa Rica la talla de mínima extracción permitida es de $47 \mathrm{~mm}$, según decreto No.13371-A del Instituto Costarricense de Pesca y Acuacultura. Esta medida constituye la primera precaución de manejo tomada para la conservación de la piangua en los manglares del país. Por ejemplo, en los manglares de Golfito, la longitud promedio de los individuos capturados $(42.51 \mathrm{~mm})$ coincide con las tallas reportadas por Pérez-Rubio (2012) en el manglar de Purruja. En ambos estudios, la longitud es menor que la talla legal estipulada; mientras que en el manglar de Playa Blanca, la longitud promedio $(48.35 \mathrm{~mm})$ de los individuos capturados si cumple con este decreto de Ley. La longitud promedio de los individuos capturados se encuentra cercana a reportes en países como Guatemala y Perú (MacKenzie, 2001). Este autor explica que el pequeño tamaño de los individuos en estos países se atribuye al poco tiempo de crecimiento por parte de los 
animales debido a la alta extracción ejercida por los usuarios del manglar. Situación similar se presenta en los manglares de Golfito; donde la alta demanda por parte de los piangüeros de la zona no permiten un crecimiento adecuado de los organismos.

La falta de alternativas económicas en la zona sur de Costa Rica, el fácil acceso a los manglares, la alta demanda de la piangua por parte de los consumidores, aunado a los pequeños tamaños de los organismos y la baja densidad, hacen que la presión sobre este recurso aumente, reflejándose en una captura menor de individuos cada año. En un estudio sobre la dinámica poblacional de $A$. tuberculosa realizado por Stern-Pirlot y Wolff (2006), corroboran una fuerte sobre pesca de esta especie en varios manglares de la costa Pacífica de Costa Rica, incluyendo los manglares de Purruja y Rincón, Golfo Dulce. Sumado a esta presión antropogénica, es importante considerar factores ambientales que pueden provocar altas temperaturas del agua o altas precipitaciones que pueden afectar el reclutamiento de juveniles lo que incrementa la probabilidad que este recurso disminuya considerablemente (Din \& Ahamad, 1995; Lucero et al., 2012; Lucero et al., 2013).

El establecimiento de programas de repoblamiento de la piangua en los manglares del Golfo Dulce, junto a proyectos de manejo y cuotas de captura, parecen ser herramientas útiles en la conservación de este recurso; sin embargo deben contar con el apoyo del gobierno y la responsabilidad de los usuarios del manglar para ser gestionadas correctamente y evitar de esta manera el peligro de extinción de A. tuberculosa.

\section{AGRADECIMIENTOS}

El artículo representa los resultados de los proyectos números 111-A2-113 y 808-A6-124 de la Universidad de Costa Rica, adscritos a la Escuela de Biología y al Centro de Investigación en Ciencias del Mar y Limnología (CIMAR). Se agradece el apoyo a los miembros de la Asociación Mixta de Piangüeros de Purruja, Golfito (APIAPU) y la Asociación
Protectores del Manglar de Playa Blanca de Puerto Jiménez (ASOMANGLE) por la colaboración durante el trabajo de campo. Fernando Mora Rodríguez realizó los mapas incluidos en este artículo. Se agradece la colaboración de Alexander Castillo, Tito Isaac Sancho, David Mena y Miguel Artavia.

\section{RESUMEN}

El presente estudio describe la dinámica de la población del bivalvo Anadara tuberculosa conocido en Costa Rica como piangua, en varios manglares de Golfito y Playa Blanca de Puerto Jiménez, Golfo Dulce en la costa Pacífica de Costa Rica. El estudio está basado en varios muestreos realizados mensualmente durante el periodo 2008-2010. A cada individuo recolectado se midió la longitud, el peso total y el peso fresco para calcular variables como el rendimiento, densidad y distribución de esta especie. La población de pianguas en los manglares de Golfito presentó mayor densidad (2.77 individuos $/ \mathrm{m}^{2}$ ) que el manglar de Playa Blanca $\left(0.14\right.$ individuos $\left./ \mathrm{m}^{2}\right)$. Se observó que los individuos de Playa Blanca presentaron mayor longitud $(48.35 \mathrm{~mm})$ que los reportados para los manglares de Golfito $(42.51 \mathrm{~mm})$, este último valor, por debajo del mínimo de extracción estipulado por Ley. Playa Blanca presentó también el mayor peso total (36.35g) que Golfito (24.48g). Este aumento del peso total en los individuos de Playa Blanca incide en que el rendimiento de la carne en este manglar resulte menor (17.01\%) que en Golfito (23.45\%). Los resultados sugieren que la población de A. tuberculosa puede encontrase en estado de reducción en estos manglares y por consiguiente, es necesario implementar proyectos de repoblamiento de este molusco en los manglares del Golfo Dulce, en conjunto con proyectos de manejo en una acción en conjunto con las autoridades gubernamentales responsables, organizaciones no gubernamentales y la Sociedad Civil, para hacer de esta especie un recurso que sea sostenible y aprovechada por diferentes actores de la comunidad.

Palabras clave: Anadara tuberculosa, manglar, Mollusca, Golfo Dulce, Costa Rica, densidad, producción.

\section{REFERENCIAS}

Ampie, C. \& Cruz, R. (1989). Tamaño y madurez sexual de Anadara tuberculosa (Bivalvia: Arcidae) en Costa Rica. Brenesia, 31, 21-24.

Borda, C. \& Cruz, R. (2004). Reproducción y reclutamiento del molusco Anadara tuberculosa (Sowerby, 1833) en el Pacífico Colombiano. Revista de Investigación Marina, 25(3), 185-195. 
Cruz, S. (1982). Variación mensual del índice de condición del molusco Anadara tuberculosa (Pelecypoda: Arcidae) en Punta Morales, Puntarenas, Costa Rica. Revista de Biología Tropical, 30, 1-4.

Cruz, R. \& Palacios, J. (1983). Biometría del molusco Anadara tuberculosa (Pelecypoda: Arcidae) en Punta Morales, Puntarenas, Costa Rica. Revista de Biología Tropical, 31, 175-179.

Cruz, R. (1984). Algunas aspectos de la reproducción en Anadara tuberculosa (Pelecypoda: Arcidae) de Punta Morales, Puntarenas, Costa Rica. Revista de Biología Tropical, 32(1), 45-50.

De la Cruz, M. (1994). Morphometric characterization and reproductive biology of Anadara tuberculosa populations from different mangrove systems located in the Nicoya Gulf, Costa Rica (Doctoral thesis). URIFE Universitait, Brussels, Belgium.

Din, Z. \& Ahamad, A. (1995). Changes in the scope for growth of blood cockles (Anadara granosa) exposed to industrial discharge. Marine Pollution Bulletin, $31,406-410$

Ellison, J. C. (1998). Impacts of sediment burial on mangroves. Marine Pollution Bulletin, 37, 8-12.

Flores, L. \& Licandeo, R. (2010). Estructura de tallas y proporción sexual de Anadara tuberculosa y Anadara similis en una reserva de manglares al noroeste de Ecuador. Revista de Biología Marina y Oceanografia, 45(3), 541-546.

Lucero, C., Cantera, J., \& Neira, R. (2012). Pesquería y crecimiento de la piangua (Arcoida: Arcidae) Anadara tuberculosa en la Bahía de Málaga del Pacífico colombiano, 2005-2007. Revista de Biología Tropical, 60(1), 203-217.

Lucero, C., Cantera, J., Gil, D., Muñoz, O., Zapata, L., Cortes, N., ... Manjarres, A. (2013). Análisis espacio temporal de la biología reproductiva y el reclutamiento del molusco bivalvo Anadara tuberculosa en la costa del Pacífico colombiano. Revista de Biología Marina y Oceanografia, 48(2), 321-334.

MacKenzie, C. (2001). The Fisheries for Mangrove Cockles, Anadara spp., from Mexico to Peru, With Descriptions of Their Habitats and Biology, the Fishermen's Lives, and the Effects of Shrimp Farming. Marine Fisheries Review, 63(1), 1-39.

Manjarrés, A. (2011). Análisis de la abundancia y madurez sexual del molusco bivalvo Anadara similis (Adams, 1852) Familia: Arcidae, en el manglar de Luisico, Archipiélago de la Plata, Bahía Málaga, Pacífico colombiano (Tesis de Maestría). Universidad del Magdalena, Colombia.
Manjarrés, A., Lucero, C., Gualteros, W., Cantera, J., \& Gil, D. (2013). Abundancia y Madurez Sexual de Anadara similis en el Manglar de Luisico, Bahía Málaga, Pacífico Colombiano. Boletín de Investigaciones Marinas y Costeras 42(2), 215-231.

Morales, A., Silva, M., \& González, C. (2009). La gestión integrada de la zona costera en Costa Rica: experiencias y perspectivas. En J. M. Barragán (Ed.), Manejo Costero Integrado y Politica Pública en Iberoamérica: Un diagnóstico. Necesidad de Cambio (pp. 40-55). España: Red IBERMAR (CYTED).

Morales, A., Silva, M., González, C., Villalobos, L., \& Ash, J. (2011). Propuestas para una gestión integrada de las áreas costeras de Costa Rica. En J. M. Barragán (Ed.), Manejo Costero Integrado y Política Pública en Iberoamérica: Propuestas para la acción (pp. 40-54). España: Red IBERMAR (CYTED).

Pérez-Rubio, I. (2012). Pesquería artesanal de Anadara tuberculosa y A. similis en el marco de una estrategia de gestión comunitaria en el manglar de Purruja (Pacífico Sur, Costa Rica) (Trabajo de comunicación). I Congreso Iberoamericano de Gestión Integrada de Áreas Litorales, España.

Silva, A. \& Carrillo, N. (2004). El manglar de Purruja, Golfito: un modelo para su manejo. Revista de Biología Tropical, 52(2), 195-201.

Silva, A. \& Bonilla, R. (2001). Abundancia y morfometría de Anadara tuberculosa y A. similis (Mollusca: Bivalvia) en el manglar de Purruja, Golfo Dulce, Costa Rica. Revista de Biología Tropical, 49(2), 315-320.

Squires, H., Esteves, M., Barona, O., \& Mora, O. (1975). Mangrove cockles, Anadara spp. of the Pacific coast of Colombia. The Veliger, 18, 57-68.

StataCorp. (2005). Software estadístico. College Station. Texas, USA.

Stern-Pirlot, A. \& Wolff, M. (2006). Population dynamics and fisheries potential of Anadara tuberculosa (Bivalvia: Arcidae) along the Pacific coast of Costa Rica. Revista de Biología Tropical, 54(1), 87-99.

Vega, A. (1994). Estructura de la población, rendimiento y épocas reproductivas de Anadara spp. (Bivalvia, Arcidae) en la Reserva Forestal Térraba-Sierpe, Puntarenas Costa Rica. Con recomendaciones para su manejo (Tesis de Maestría). Universidad de Costa Rica, Costa Rica. 